\title{
THE MUENSTER REDSHIFT PROJECT (MRSP)
}

\author{
P. SCHUECKER, W.C. SEITTER, P. BOSCHAN, R. BUDELL, B. CUNOW, \\ R. DUEMMLER, M. NAUMANN, H.-A. OTT, G. SPIEKERMANN, D. TEUBER \\ and R. UNGRUHE \\ Astronomisches Institut \\ Universität Münster, Germany
}

\begin{abstract}
The Muenster Redshift Project provides to date 0.9 million low-resolution redshifts obtained from automatic reductions of pairs of direct and low-dispersion objective prism Schmidt plates. Preliminary results obtained from subsamples of the survey are described.
\end{abstract}

\section{Introduction}

Two requirements must be fulfilled by galaxy catalogues used in cosmological investigations: 1) large numbers of galaxies in 2) large volumes. An efficient observational strategy to meet both requirements is to measure the redshifts $z$ from large field objective prism plates with dispersions permitting to go as deep as possible while the wavelength resolution is still acceptable. This procedure significantly reduces the observing time, which is otherwise the longest and most costly part of the measuring process. In the Muenster Redshift Project (MRSP, Seitter 1992) $z$-values are determined for normal galaxies with magnitudes $m<20$ and $z \leq 0.3$ from objective prism Schmidt plates of linear reciprocal dispersion $246 \mathrm{~nm} / \mathrm{mm}$ at $\mathrm{H}_{r}$. The spectra give redshifts with random errors $\sigma(z)=0.012$ for more than $75 \%$ of the galaxies (Schuecker 1993). Extensive simulations show that this resolution suffices to study a number of important cosmological problems.

This paper reviews the present status of the survey and illustrates the use of low-resolution data in cosmology by describing the determination of three basic cosmological quantities: the variances $\sigma^{2}(L)$ on scales $L$ and the power spectrum $P(k)$ of galaxy number density fluctuations with wavelengths 10 to $160 \mathrm{Mpc}\left(H_{0}=100 \mathrm{~km} / \mathrm{sec} / \mathrm{Mpc}\right)$, the Hubble constant $H_{0}$, and the deceleration parameter $q_{0}$. The three examples are exemplary for three different ways of using low-resolution data:

1) the values of $\sigma^{2}(L)$ and $P(k)$ are corrected for redshift errors and checked against data from other surveys and numerical models;

2) for the determination of $H_{0}$ a model luminosity function, based on high quality empirical data, is convolved with the MRSP redshift errors for comparison with the measured low-resolution data;

3) the measurement of $q_{0}$ is nearly unaffected by redshift errors, and on the present noise level, redshift corrections are not necessary. 


\section{Present Status of the Catalogues}

The determination of redshifts relies to a large degree on the proper reduction of the corresponding direct Schmidt plates. On the direct plates, the star/galaxy separation (Horstmann 1992), the magnitude measurements (Cunow 1993a) and the morphological classifications of the galaxies according to Hubble types (Spiekermann 1992) are carried out. All data are obtained from film copies digitized with the two measuring machines PDS2020GMplus at Münster. We find no significant positional differences between film and glass copies. Concerning magnitude measurements it is indicated that accuracies depend more on the measuring machines than on the photographic medium (Sommer 1993). Fong (1993) showed that double slit machines (e.g. PDS) give best accuracy.

Two direct catalogues are presently available:

1) ESO IIIa-F plates (Naumann, Ungruhe \& Seitter 1993): $7.1 \cdot 10^{6}$ galaxies, red magnitudes $r_{F} \leq 21.0$ in $5100 \square^{\circ}$ centred at the South Galactic Pole SGP, CCD sequences for magnitude calibrations (Cunow 1993b, 1993c);

2) ESO/SERC IIIa-J plates (Horstmann 1992): $6.5 \cdot 10^{5}$ galaxies, blue magnitudes $b_{J} \leq 21.0$ in $400 \square^{\circ}$ near the SGP, CCD sequences for magnitude calibrations (Cunow 1993a).

The two surveys yield the following catalogues:

$0.9 \cdot 10^{6}$ redshifts in $750 \square^{\circ}$ for galaxies with $r_{F} \leq 20.5$ (input catalogue: IIIa-F);

$1.2 \cdot 10^{5}$ redshifts in $300 \square^{\circ}$ for galaxies with $b_{J} \leq 19.8$ (input catalogue: IIIa-J) (Sample A);

$1.0 \cdot 10^{6}$ morphological types in $1300 \square^{\circ}$ (input catalogue: IIIa-F);

$1.0 \cdot 10^{5}$ morphological types in $400 \square^{\circ}$ (input catalogue: IIIa-J);

$2.9 \cdot 10^{3}$ quasar candidates in $300 \square^{\circ}$ (input catalogue: IIIa-J).

The accuracy of the subsequent investigations rely on the correct determination of the magnitude zero points. Presently, the magnitude calibration is completed for the catalogues based on IIIa-J, thus, redshift sample A is used in the following.

\section{Fluctuations of Galaxy Number Densities}

In order to avoid the use of magnitude- and redshift-dependent selection functions, a volume- and absolute magnitude-limited subsample is selected: 3544 galaxy redshifts with $0.11<z<0.21$, $17<b_{J}<19.3$ and absolute magnitudes $-21.5<M<-20.4$. Fluctuation analysis is performed in comoving space with the standard values of the cosmological parameters $\Lambda=0, \Omega_{0}=2 q_{0}=1$ and $K$-correction $K(z)=3 \cdot z[\mathrm{mag}]$ for all galaxy types.

In extension to earlier fluctuation studies (Schuecker \& Ott 1991), cell count analysis is applied, whereby galaxies are counted in cubic boxes with length $L$, shifted by tessellation over the survey volume. From the measured variances the discreteness noise (Poisson) is subtracted. The errors are estimated analytically including the measured inhomogeneities of galaxy density, assuming a Gaussian random field (Efstathiou et al. 1990). This assumption is (partially) verified by estimating the third- and fourth-order moments of the galaxy distributions. They indicate that no large deviations from zero are present for $L>30 \mathrm{Mpc}$. The measured variances are corrected 
for the redshift errors using

$$
P(k)_{\text {obs }}=P(k)_{c o r} \frac{\sqrt{\pi}}{2} \cdot \frac{\operatorname{erf}\left(\frac{c}{H_{0}} \sigma_{z} k\right)}{\frac{c}{H_{0}} \sigma(z) k}
$$

where $P(k)_{o b s}$ and $P(k)_{c o r}$ are the observed and corrected power spectrum, respectively (Peacock 1992). The transformations of the variances into $k$-space (and back to real space) are obtained at the effective wavenumbers $k_{\text {eff }}=2 \pi / \lambda_{\text {eff }}$ using

$$
\sigma^{2}\left(L\left(\lambda_{e f f}\right)\right) \approx \frac{2}{\pi^{2}} k_{e f f}^{3} P\left(k_{e f f}\right)
$$

(Peacock 1991), with the effective wavelength $\lambda_{\text {efr }}$ For power spectra with spectral index $n \leq 0$ a good estimation of the effective wavelength is $\lambda_{\text {eff }}=2 L$. The corrected variances are shown in Fig. 1a. They are compared with the measurements obtained for IRAS galaxies and with the (standard) CDM model, both given in Efstathiou et al. (1990). On all scales, no significant differences between the two observed variances and the variances expected for the CDM model are seen. The present data do not rule out the CDM model.

For $\lambda_{\text {eff }} \leq 80 \mathrm{Mpc}$ the corrected power spectrum (Fig. 1b) has the spectral index $n=-1.3_{-0.6}^{+0.2}$. On large scales indications of a turnover in the power spectrum are seen. Measurements including the total redshift sample are now in progress. They will permit fluctuation measurements for $\lambda_{\text {eff }}=300 \mathrm{Mpc}$ with significantly reduced statistical errors.
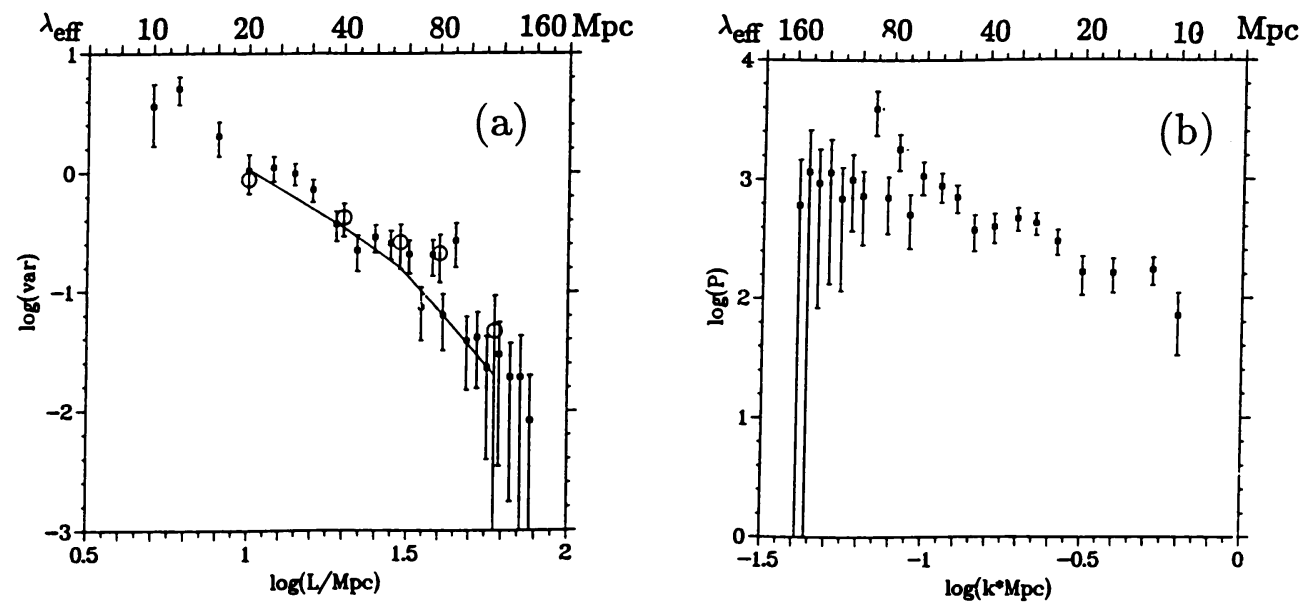

Figure 1. a) Variances of comoving fluctuations of galaxy number densities, corrected for $z$-errors, b) power spectrum of effective wavelengths, corrected for $z$-errors. MRSP data: boxes; IRAS data: circles; line: standard CDM model. 


\section{Hubble Constant $\boldsymbol{H}_{0}$}

In order to determine the Hubble constant from local peculiar velocity effects, i.e. at relatively large $z$, an apparent luminosity function $\Phi\left(m\left(z_{r}\right)\right)$, obtained at large redshifts, is compared with a reference LF $\Phi\left(m_{M}\right)$. The apparent reference LF $\Phi\left(m_{M}\right)$ is created by Monte Carlo simulations, based on the observed apparent magnitudes of nearby galaxies, convolved with the measuring errors of the MRSP sample, and transformed to their apparent magnitudes $m_{M}$ at $z_{r}$. For the last step the luminosity distances are computed from the Mattig formula with $q_{0}=0.5$, using the best available distance modulus of the local galaxies and different values of $H_{0}$ which must be varied to give the best fit to the two LFs. Figure 2a shows the LF of 63,151 MRSP galaxies with $K$ corrected total magnitudes $b_{J}$, in the range $0.05 \leq z \leq 0.2$, transformed to the reference redshift $z_{r}=0.1$, together with the reference luminosity function based on 320 galaxies of the Virgo cluster at best fit. Dwarf galaxies are excluded from the Virgo sample since they are not observed in the MRSP sample. The shapes of the two LFs are sufficiently similar to justify the procedure. Results from 12 fields yield $35<H_{0}<55 \mathrm{~km} / \mathrm{s} / \mathrm{Mpc}$ for $30.9<(m-M)<31.7$ of the Virgo cluster (Duemmler 1992, 1993).

\section{Deceleration Parameter $q_{0}$}

For the determination of $q_{0}$ the classical redshift-volume test (e.g. Robertson \& Noonan 1968; Loh \& Spillar 1986) is used. In the present version of the test, the measured comoving galaxy number densities $n(z)$ are compared with the flat density plateaux, expected for the correct $q_{0}$. If the values of the deceleration parameter used for the calculation of the $n(z)$ are wrong, systematic slopes are introduced into the measured density distributions.
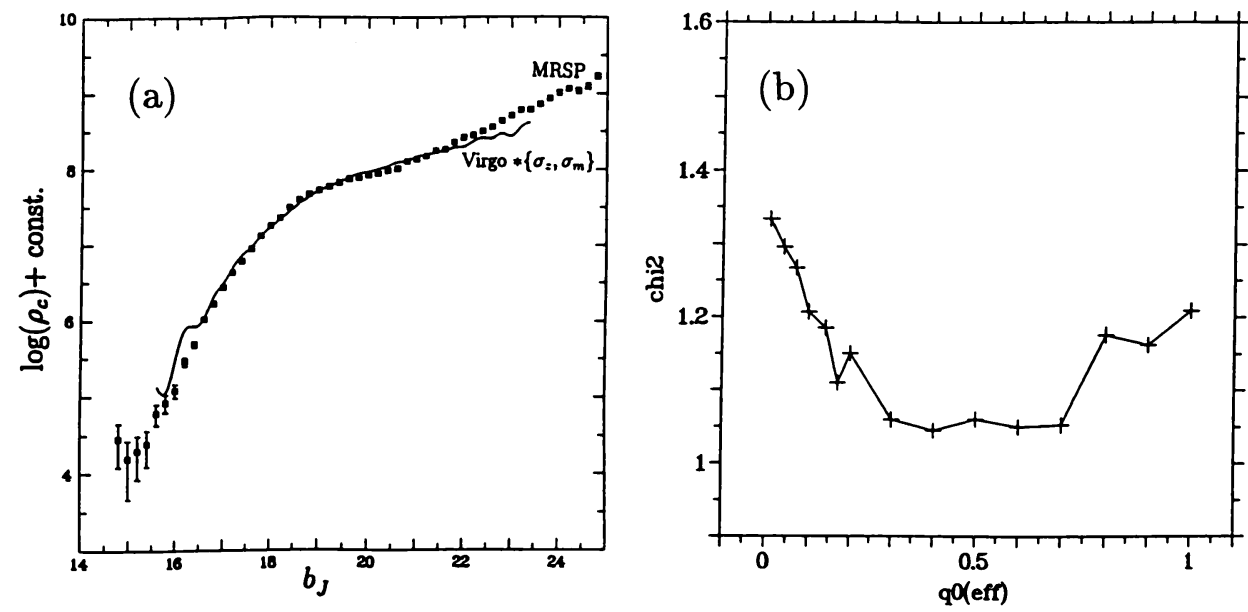

Figure 2. a) Determination of $H_{0}$ : comparison of the apparent MRSP luminosity function, where all magnitudes are transformed to the reference redshift $z_{r}=0.1$ (boxes) and the reference LF based on the Virgo cluster without dwarf galaxies, convolved with the measuring errors of the MRSP sample (line). b) Determination of $q_{0}: \chi^{2}$-values for different $q_{0}$ as obtained from the redshift volume test. For details see text. 
The major advantage of the test is that all matter, luminous and dark, as well as the cosmological constant, contribute to the growth of volumes with $z$, and the value of $q_{0}$, thus determined, includes all possible effects. Two problems of the test are: 1) galaxy evolution, 2) counting noise.

1) In order to reduce the influence of possible galaxy evolution, the redshift and absolute magnitude ranges are restricted to $0.1<z<0.2$ and $-21.5<M<-20.5$, respectively. Indications that significant global evolutionary effects are not present in the MRSP sample, come from differential galaxy number counts $N\left(b_{J}\right)$ obtained from the IIIa-J direct plates. Preliminary tests show that the number counts within the magnitude range $17<b_{J}<20$ are well-described by the standard LF $\left(M_{*}=-19.68, \alpha=-1.0\right), K$-correction $K(z)=3 z, q_{0}=0.5$ without number density or luminosity evolution. To support this, additional tests using the frequency distribution of colours $b_{J}-r_{F}$ for several million galaxies of different morphological types are in preparation.

2) The problem of counting noise is reduced by the many redshifts - such as are obtained from a low-resolution objective prism redshift survey. For the present study about 3,000 galaxy redshifts are used, and Monte-Carlo simulations show that this number is large enough to illustrate the principle effects of $q_{0}$. The determination of $q_{0}$ with more than 10,000 galaxies is in preparation.

To test the flatness of the $n(x)$ distributions, a $\chi^{2}$ test is applied. In Fig. $2 b$ the $\chi^{2}$ values for different $q_{0}$ are shown, yielding $q_{0}=0.5 \pm 0.2$ from the present sample.

\section{Acknowledgements}

The provision of film copies of spectral plates by the UK-Schmidt Telescope Unit, Edinburgh, is gratefully acknowledged. The MRSP is partially supported by the Deutsche Forschungsgemeinschaft under Se 345/14-1,2,3.

\section{References}

Cunow, B., 1993a. Astron. Astrophys. Suppl., 97, 541.

Cunow, B., 1993b. Astron. Astrophys., 268, 491.

Cunow, B., 1993c. Astron. Astrophys. Suppl., in press.

Duemmler, R., 1992. Astron. Astrophys., 264, 1.

Duemmler, R., 1993. PhD thesis, Univ. Münster (in preparation).

Efstathiou, G., Kaiser, N., Saunders, W., Lawrence, A., Rowan-Robinson, M., Ellis, R.S. and Frenk, C.S., 1990. Mon. Not. R. astron. Soc., 247, 10p.

Fong, R., 1993. These proceedings.

Horstmann, H., 1992. PhD. thesis, Univ. Münster.

Loh, E.D. and Spillar, E.J., 1986. Astrophys. J., 307, L1.

Naumann, M., Ungruhe, R. and Seitter, W.C., 1993. ESO Messenger, 71, 46.

Peacock, J.A., 1991. Mon. Not. R. astron. Soc., 253, 1 p.

Peacock, J.A., 1992. In Proc. Valencia Summer School, New Insights into the Universe, ed. V.J. Martinez, Springer-Verlag, Dordrecht, p. 212. 
Robertson, H.P. and Noonan, T.W., 1968. In 'Relativity and Cosmology', W.B. Saunders Company, Philadelphia, p. 358.

Schuecker, P., 1993. Astrophys. J. Suppl., 84, 39.

Schuecker, P. and Ott, H.A., 1991. Astrophys. J. Lett., 378, L1.

Seitter, W.C., 1992. In 'Digitised Optical Sky Surveys', eds. H.T. MacGillivray and E.B. Thomson, Kluwer Academic Publishers, Dordrecht, p. 367.

Sommer, R., 1993. In preparation.

Spiekermann, G., 1992. Astron. J., 103, 2102. 